\title{
Uma experiência educacional de incentivo ao aleitamento materno e estimulação do bebê, para mães de nível sócio-econômico baixo: estudo preliminar 1
}

\author{
An education experience for promoting \\ breast-feeding and infant stimulation by \\ low-income women: a preliminary study 1
}

Victoria Garcia-Montrone 2

Júlio C. de Rose 3

\footnotetext{
1 Artigo baseado

na dissertação de Mestrado

da primeira autora,

apresentada ao Programa de

Pós-Graduação em Educação

Especial da Universidade

Federal de São Carlos.

2 Programa de Pós-Graduação

em Educação, Centro de

Educação e Ciências Humanas,

Universidade Federal de São

Carlos.

C.P. 676, São Carlos, SP

13565-905, Brasil.

3 Departamento de Psicologia,

Universidade Federal de

São Carlos

C.P. 676, São Carlos, SP

13565-905, Brasil.
}

Resumo Este estudo efetuou uma avaliação preliminar da eficácia de um programa educacional de estímulo ao aleitamento materno, que inclui a introdução simultânea de técnicas de estimulação do bebê. O programa, elaborado por Garcia-Montrone (1992), foi aplicado a um grupo de mães de nível sócio-econômico baixo (grupo experimental). Tal programa foi avaliado através de sessões de observação direta da interação mãe-criança e entrevistas com as mães até seis meses após o nascimento do bebê, comparando-se os resultados com os de um grupo-controle com características similares de idade, nível educacional e experiência prévia em amamentação. Cada grupo continha inicialmente dez diades, havendo um abandono em cada grupo durante o estudo. Os resultados mostraram que 55\% das mães do grupo experimental ainda amamentavam seus filhos aos seis meses, contra apenas $22 \%$ daquelas do grupo-controle. Em relação à estimulação do bebê, o grupo experimental mostrou uma freqüência significativamente maior de episódios de estimulação que o grupo-controle. Os autores concluem que é factível incluir simultaneamente aleitamento materno e estimulação do bebê num programa educacional para mulheres de nível sócio-econômico baixo e que a aplicação deste programa pode contribuir para reduzir o risco de desnutrição e retardo do desenvolvimento do bebê.

Palavras-chave Aleitamento Materno; Educação em Saúde; Saúde da Criança

Abstract This study included a preliminary analysis of the effectiveness of an educational program on breast-feeding and infant stimulation techniques. The program, developed by GarciaMontrone (1992), was applied to a group of mothers (experimental group) of low socio-economic status. The program was evaluated by direct observation of mother-infant interaction and interviews with the mothers up to six months after childbirth. Results were compared with a control group of women matched for age, educational level, and previous experience with breast-feeding. Each group began with 10 mother-infant dyads, and there was one withdrawal in each group over the course of the study. 55\% of the mothers in the experimental group were still breast-feeding six months after childbirth, compared to $22 \%$ in the control group. The experimental group also showed a significantly higher frequency of stimulation of the infant, both during and after breast-feeding. The authors conclude that it is feasible to combine breast-feeding and infant stimulation instruction in an educational program for women of low socio-economic status and that the use of this program can help reduce risk factors both for malnutrition and developmental retardation.

Key words Breast Feeding; Health Education; Children's Health 


\section{Introdução}

Tanto o aleitamento materno quanto a estimulação adequada do bebê constituem fatores fundamentais para o desenvolvimento nutricional, motor, cognitivo e psicossocial das crianças, principalmente nos primeiros meses de vida.

O declínio acentuado no aleitamento materno, ocorrido sobretudo no mundo ocidental, depois da Primeira Guerra Mundial, constitui um problema sério, por suas graves conseqüências para a saúde (morbidade, desnutrição e mortalidade infantil). Este declínio na amamentação se evidencia tanto no que se refere a um número cada vez menor de mães dispostas a amamentar, quanto ao início, cada vez mais precoce, do desmame. No Brasil, os índices de amamentação eram considerados bons até a década de 60 , constatando-se um sensível declínio na prática e na duração do aleitamento materno na década seguinte, seguindo a tendência internacional (Tudisco et al.; 1984, Vinha \& Scochi, 1989). Um estudo realizado no sul do Brasil revelou que, em 1940, 98\% dos bebês se alimentavam quase que exclusivamente no peito, enquanto que, em 1974, este valor atingia apenas $76 \%$. Além disto, a porcentagem de mães que amamentavam seus filhos aos seis meses diminuiu de $60 \%$ para $12 \%$ (RodríguezGarcia \& Schaefer, 1991).

A lactância natural constitui a melhor opção do lactante menor de seis meses, pela sua superioridade nutricional, defesa imunológica e ausência de agressão físico-química (Hardy et al., 1982, 1987; Juez, 1989; Vinha, 1988). Por este motivo, o desmame precoce, especialmente quando associado a condições de miséria e insalubridade, aumenta a morbi-mortalidade das crianças. Verificou-se que, quanto menor o tempo de amamentação, maior é a incidência de hospitalizações, de desnutrição e de mortalidade infantil (Miura, 1978; Victora et al., 1989). Vários estudos concluíram que estes fatores, especificamente nas classes sócio-econômicas mais baixas, são decorrentes de alimentação inadequada e baixo nível de saneamento ambiental, e ressaltam a importância da promoção do aleitamento materno nesta população de risco (e.g., Gulla \& Benech, 1984; Martins Filho, 1985).

As pesquisas apontam que os principais fatores de desmame precoce podem ser classificados em dificuldades circunstanciais (mamilos achatados, fissuras, etc.) e em dificuldades culturais. Entre estas últimas, podemos citar: a transformação ocorrida na estrutura familiar, sobretudo nas sociedades urbanas; os diversos papéis desempenhados pela mulher na sociedade moderna; a apresentação das mamas como símbolo sexual, contribuindo, juntamente com outros hábitos sociais, para que as mulheres não gostem de amamentar em público; a propaganda de produtos lácteos para alimentação de bebês e a perda da tradicional transmissão de mãe para filha da experiência em amamentação (Issler, 1987). Podem ser acrescentados a estes fatores, práticas dos serviços de maternidade, como a demora em levar o bebê ao seio após o nascimento, a sedação do recém-nascido em função do excesso de anestesia materna e a administração de chás, água glicosada ou alimento complementar (Moura \& Santoro, 1980).

Diante destas dificuldades, é necessário propor estratégias centradas no aspecto educativo, abrangendo tanto a difusão de informações a respeito da importância e das vantagens do aleitamento materno, como também a instrução das mães a respeito da forma correta de amamentar, das técnicas específicas para superar as dificuldades circunstanciais que podem ocorrer principalmente no início da amamentação e das técnicas especiais para compatibilizar a amamentação com os outros papéis desempenhados pela mulher dentro da sociedade.

Muitos programas bem-sucedidos de incentivo ao aleitamento materno têm sido desenvolvidos nos últimos anos, no sentido de esclarecer sobre a importância do aleitamento e ensinar como fazê-lo (Granzoto et al., 1992; Hardy et al., 1982, 1987; Siqueira et al., 1994; Vinha, 1988). O sucesso destes programas mostra que as populações-alvo respondem favoravelmente a uma intervenção educacional.

Nosso estudo, além da instrução sobre aleitamento materno, introduziu simultaneamente o ensino de técnicas de estimulação do bebê, na situação de aleitamento, considerando que muitos autores destacam a importância da estimulação para as crianças, sobretudo aquelas pertencentes a grupos de risco (Brazelton, 1981; Sameroff \& Fiese, 1990; Windholz, 1988). Estes estudos destacam o impacto dos programas de estimulação na prevenção do atraso no desenvolvimento destas crianças. Estes programas de estimulação devem ser aplicados o mais cedo possível para maximizar sua eficácia. Seus benefícios consistiriam basicamente em ganhos no desenvolvimento cognitivo, de linguagem, motor e sócio-emocional. Neste sentido, a O. P. S. (1988) afirma que a falta de estimulação numa criança sadia pode limitar seu desenvolvimento.

A mãe é o principal agente de estimulação do bebê, já que é ela que tem contatos mais 
freqüentes com ele. $\mathrm{O}$ ato de amamentar propicia contato direto entre a mãe e o bebê, constituindo-se numa oportunidade de interação que favorece o estabelecimento de vínculos afetivos, os quais são indispensáveis para o desenvolvimento afetivo-emocional e social ao longo de toda a infância (Bowlby, 1976; Rodriguez-Garcia \& Schaefer, 1991). Mais ainda, o ato de amamentar se constitui numa boa oportunidade para aplicar técnicas de estimulação que venham favorecer o desenvolvimento integral do bebê. Durante a amamentação, a mãe pode, entre outras coisas, olhar, acariciar e conversar com seu bebê, em repetidas oportunidades todos os dias. No entanto, cabe ressaltar que nem sempre a mãe está preparada para realizar estas atividades ou sabe de sua importância para o desenvolvimento de seu bebê.

O objetivo desta pesquisa foi avaliar a eficácia de um programa educacional abrangendo simultaneamente aleitamento materno e estimulação do bebê. A avaliação da eficácia do programa envolveu, além de entrevistas com as mães, observações diretas no domicílio das participantes, durante seis meses após o nascimento do bebê. O programa educacional foi destinado a uma população de nível sócio-econômico baixo, considerada de risco devido à incidência de fatores que contribuem para a morbi-mortalidade infantil ou atraso no desenvolvimento.

\section{Metodologia}

Esta pesquisa utilizou um delineamento experimental de comparação de grupos, sendo um experimental e o outro de controle. Para a composição dos grupos, foram identificados os dois postos de saúde com maior número de consultas pré-natais em uma cidade de porte médio do interior do Estado de São Paulo. Todas as gestantes, com 17 a 29 semanas de gravidez, agendadas nestes postos foram convidadas a participar da pesquisa. De um total de 40 convidadas, 31 aceitaram participar e foram informadas de que poderiam, conforme distribuição a ser feita posteriormente, participar de um curso sobre aleitamento materno e estimulação do bebê, ministrado no posto de saúde, ou de um curso sobre cuidados gerais com o bebê, ministrado de forma individual no domicílio. Nesta ocasião, as mães também foram informadas de que seriam observadas periodicamente no domicílio até o bebê completar seis meses.

Para a composição final dos grupos, foram eliminadas cinco gestantes que tinham mais do que três meses de experiência em amamentar seus filhos. As 26 restantes foram divididas em dois grupos, aleatorizando-se as participantes quanto às variáveis idade, escolaridade e experiência, ou não, em amamentação.

A Tabela 1 resume os dados utilizados na aleatorização dos grupos experimental e controle. Na variável idade foram considerados três grupos, incluindo mães com: (1) 14 a 18 anos, (2) 19 a 34 anos e (3) 35 anos ou mais. A média de idade das mães foi aproximadamente $23(s=6,5)$ e $24(s=7,5)$ anos, para os grupos experimental e controle, respectivamente. No que se refere à escolaridade, consideramos duas categorias: escolaridade baixa e alta, com, respectivamente, $76,9 \%$ e $23,1 \%$ das mães, em ambos os grupos. Na categoria escolaridade baixa, estão as mães que não completaram a 4a série (incluindo as analfabetas), enquanto que, na de escolaridade alta, estão aquelas que concluíram a 4 a série ou mais. Na variável experiência em amamentação também foram consideradas duas categorias: as mães sem qualquer experiência e aquelas com alguma experiência anterior, desde que não superior a três meses (as mães com experiência superior a três meses em amamentação não foram incluídas na pesquisa). Tanto no grupo experimental como no grupo-controle, 53,8\% das mães já possuíam alguma experiência anterior em amamentar.

O programa educacional, elaborado por Garcia-Montrone (1992), foi aplicado ao grupo experimental, composto por doze mães (houve uma desistência), em cinco sessões de, aproximadamente, uma hora cada, num ritmo de uma sessão por semana. Tais encontros foram realizados num posto de saúde da cidade. $\mathrm{Ne}$ les, procuramos ensinar os conhecimentos e

Tabela 1

Distribuição de sujeitos nos grupos experimental e controle quanto às variáveis de aleatorização.

\begin{tabular}{lcc}
\hline & experimental & controle \\
\hline Primigesta & 5 & 5 \\
Multípara & 8 & 8 \\
Idade 1 (14 a 18) & 3 & 3 \\
Idade 2 (19 a 34) & 9 & 9 \\
Idade 3 (35 ou mais) & 1 & 1 \\
Escolaridade baixa & 10 & 10 \\
Escolaridade alta & 3 & 3 \\
Sem experiência anterior em amamentação & 6 & 6 \\
Com experiência anterior em amamentação & 7 & 7 \\
\hline
\end{tabular}


habilidades necessários para atingir os objetivos do programa educacional, a saber, (1) amamentar o bebê exclusivamente no seio até no mínimo seis meses após o parto, utilizando os conhecimentos e técnicas aprendidas no programa de ensino e (2) estimular adequadamente o bebê, ou seja, emitir os comportamentos recomendados para estimular o bebê e responder às iniciativas apresentadas por ele, na situação de aleitamento.

Cada sessão para aplicação do programa era composta das seguintes fases: (A) revisão da sessão anterior, para verificar a fixação dos conhecimentos adquiridos. Nesta fase, se fossem percebidas dificuldades, a professora (a primeira autora deste trabalho) começava pela reapresentação e/ou demonstração do aspecto em questão. A fase (B) consistia numa exposição da professora, apresentando também uma seqüência de diapositivos. Na fase (C), as mães recebiam um folheto com exercícios e/ou explicações, o qual era lido integralmente por uma das participantes. A fase (D) consistia na demonstração dos exercícios ou técnicas, com base no folheto distribuído. Para estas demonstrações, foi utilizado, quando necessário, um modelo de mamas, chamado "seio-cobaia" (Campestrini, 1987). Na fase (E), cada mãe realizava os exercícios no "seio-cobaia”. Depois que cada participante fazia o exercício, ela recebia feedback a respeito de sua execução. A professora, em geral, deixava primeiro que as colegas comentassem sobre o exercício realizado pela participante, e, em seguida, quando necessário, fazia seus próprios comentários. Ela também incentivava as participantes a realizarem os exercícios em casa, nos próprios seios, com o auxílio do folheto. A fase (F), a última, consistia em uma discussão em grupo sobre os pontos importantes da sessão e sobre a experiência prévia das mães a respeito dos tópicos apresentados. O objetivo desta fase era verificar se as mães tinham adquirido os conhecimentos tratados na sessão.

As participantes do grupo de controle receberam orientações sobre cuidados gerais com o bebê logo após o nascimento do mesmo. Estas orientações foram dadas no domicílio, em uma sessão de aproximadamente 1 hora, e incluíram cuidados higiênicos, curativos do coto umbilical e vacinas. As orientações a estas mães não incluíram alimentação e estimulação do bebê. As participantes de ambos os grupos foram orientadas a continuar com o atendimento de rotina oferecido no posto de saúde mais próximo de sua residência.

A coleta de dados visando à avaliação do programa educacional foi realizada no domicí- lio das mães, por observadoras independentes. As observadoras eram todas estudantes de pósgraduação, que concordaram em colaborar com a pesquisa. A primeira autora discutiu com elas os objetivos da pesquisa e a necessidade de que desconhecessem o grupo a que pertenciam as mães observadas. As observadoras foram solicitadas a não conversar sobre o assunto com as mães e informar à pesquisadora se eventualmente uma das mães mencionasse espontaneamente o curso que havia recebido. Em uma única ocasião, uma das observadoras comunicou que havia sido informada espontaneamente pela mãe sobre o curso de que havia participado. Neste caso, foi providenciada a troca da observadora para aquela mãe.

O treinamento das observadoras foi realizado individualmente, em postos de saúde da cidade, através da observação de mães nãopertencentes aos grupos do estudo, amamentando seus filhos. Considerava-se que a observadora estava treinada quando o seu registro de observação atingia um índice de fidedignidade point by point de no mínimo 90\%. Nesta ocasião, as observadoras recebiam endereços das mães e cronograma das observações e entrevistas que deveriam realizar.

A avaliação consistiu em oito sessões de observação e entrevistas, realizadas aos sete, quinze e trinta dias de vida do bebê e depois uma vez por mês. O registro de observação referia-se a comportamentos previamente definidos de interação mãe-criança e foram colhidos em dois momentos: durante o ato de aleitamento, seja este no seio ou na mamadeira, e logo após o aleitamento, até que o bebê fosse colocado no berço ou que houvesse interrupção da interação com a mãe. O tempo máximo de observação foi de trinta minutos. Adotou-se a técnica de registro de ocorrências de categorias de comportamento por unidade de tempo (30 segundos). As categorias definidas neste trabalho estão apresentadas na Tabela 2 .

A entrevista, contendo oito questões, coletava dados sobre o tipo de aleitamento, sobre o estado de saúde geral do bebê e, em caso de desmame, sobre o motivo deste (quando, por que e quem indicou). Esta entrevista era realizada logo após o registro de observação.

Foram realizados três registros de fidedignidade para cada mãe participante do estudo, sendo o primeiro deles numa das três sessões iniciais, e os restantes na quinta e oitava sessões, respectivamente. Nestas sessões, foram feitos registros independentes por duas observadoras. $\mathrm{O}$ índice de fidedignidade foi calculado point by point utilizando a relação: $\mathrm{nc} /(\mathrm{nc}+\mathrm{nd})$ * 100 , onde nc e nd indicam o número de concor- 
Descrição das categorias de interação mãe - criança estabelecidas para observação.

\section{Categorias}

1) mãe olha o bebê

A mãe olha em direção ao rosto do bebê, fixando o olhar durante 3 segundos.

2) bebê olha para a mãe

O bebê olha em direção ao rosto da mãe, fixando o olhar durante 3 segundos.

3) mãe sorri para o bebê

A mãe sorri, tendo o seu rosto em direção ao do bebê.

4) bebê sorri para a mãe

O bebê sorri, tendo o rosto em direção ao da mãe.

5) mãe acaricia o bebê

A mãe toca partes do corpo do bebê (mão, rosto, etc) de uma forma suave, movimentos de vai e vem.

6) bebê reage à carícia da mãe

O bebê acaricia a mãe, movimenta-se, sorri, olha em direção à face da mãe, logo após a carícia dela.

7) mãe conversa com o bebê

A mãe fala, emite sons, canta, tendo seu rosto em direção ao do bebê.

8) bebê emite sons

O bebê emite sons fixando o olhar no rosto da mãe.

dâncias e de discordâncias registradas, respectivamente. A média de fidedignidade obtida foi de $96,07 \%$, com variações entre $84,3 \%$ a $100 \%$ para o grupo experimental, e de $96,09 \%$, com variações entre $92 \%$ a $100 \%$ para o grupo-controle. Apenas uma vez foi registrado um índice de fidedignidade inferior a 90\%, tendo este índice ocorrido na primeira avaliação de fidedignidade feita com uma das observadoras. Esta observadora foi retreinada em seguida, e os índices subseqüentes passaram a ser similares aos das demais observadoras.

\section{Resultados}

Das treze participantes selecionadas para o grupo experimental, uma desistiu antes do início da aplicação do programa e uma desistiu antes do início das avaliações. Outra participante deste grupo foi eliminada da amostra porque teve parto prematuro. A fase de avaliação foi iniciada com 10 participantes, tendo uma delas desistido após a sexta sessão. O grupo-controle teve duas desistências antes do início das avaliações e uma participante eliminada da amostra devido a parto prematuro. Outra participante desistiu após a segunda sessão de avaliação.
Tabela 3

Porcentagem de mães amamentando nos grupos experimental e controle.

\begin{tabular}{lcl}
\hline Idade do bebê & experimental & controle \\
\hline 7 dias & $100 \%(n=10)$ & $90 \%(n=10)$ \\
15 dias & $100 \%(n=10)$ & $80 \%(n=10)$ \\
1 mês & $100 \%(n=10)$ & $55 \%(n=9)$ \\
2 meses & $90 \%(n=10)$ & $44 \%(n=9)$ \\
3 meses & $90 \%(n=10)$ & $44 \%(n=9)$ \\
4 meses & $80 \%(n=10)$ & $44 \%(n=9)$ \\
5 meses & $66 \%(n=9)$ & $22 \%(n=9)$ \\
6 meses & $55 \%(n=9)$ & $22 \%(n=9)$ \\
\hline
\end{tabular}

Os resultados (Tabela 3) mostraram que $100 \%$ das mães do grupo experimental estavam amamentando no primeiro mês de vida do bebê e $55 \%$ continuavam amamentando os filhos aos seis meses. No grupo-controle, apenas $22,2 \%$ continuavam amamentando no quinto e sexto mês de idade do bebê. Estes resultados indicam que no grupo experimental o desmame foi menor e ocorreu tardiamente em relação ao grupo-controle.

No que se refere à estimulação do bebê, as figuras 1 e 2 mostram a média do total de ocorrências das categorias de estimulação, observadas durante e após o aleitamento, em função da idade do bebê, para ambos os grupos, experimental e controle. Foram considerados apenas os registros com dados de pelo menos cinco díades. Por este motivo, a Figura 2 não apresenta os dados do grupo experimental no terceiro registro (primeiro mês de idade do bebê), uma vez que a maioria dos bebês dormiu durante a amamentação. Nesta análise não foram incluídas as freqüências das categorias 1, 6 e 8 . A categoria 1 foi excluída porque apresentou um valor constante e idêntico para ambos os grupos, e as categorias 6 e 8 foram excluídas por terem um número muito baixo de ocorrências.

Tanto durante quanto após o aleitamento, é notável a diferença entre o grupo experimental e o grupo-controle, após o terceiro registro de observação, ou seja, quando o bebê estava com mais de um mês de idade. Foi realizada uma Análise de Variância, em modelo linear geral (Neter \& Wasserman, 1974), que mostrou que a diferença entre as médias foi significativa a um nível menor que $0,1 \%$. 
Figuras

1) Média de ocorrências das várias categorias de estimulação observadas durante o aleitamento para os grupos experimental e controle (dados normalizados para 1 minuto de observação).



2) Média de ocorrências das várias categorias de estimulação, observadas após o aleitamento, para os grupos experimental e controle (dados normalizados para 1 minuto de observação).

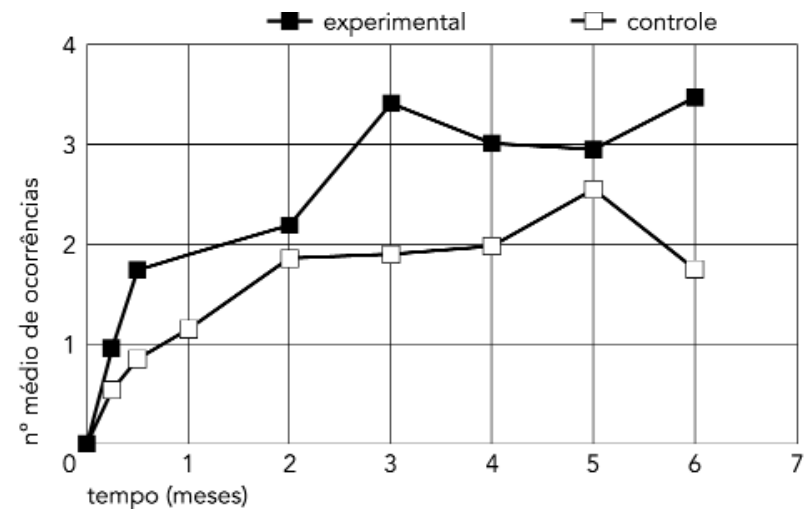

\section{Discussão}

Tendo em vista o reduzido número de díades em cada grupo (nove ao final do estudo), os resultados devem ser tomados com cautela. Os resultados de amamentação indicam que as mães do grupo experimental responderam favoravelmente ao programa educacional visando a incentivar a prática do aleitamento materno. Neste programa as mães aprenderam a importância que o aleitamento materno representa para o crescimento de seu filho, assim como a maneira correta de amamentar. Este estudo indica que programas educacionais que fornecem instruções de fácil aplicação apresentam resultados significativos. Neste sentido, podemos dizer que as mães do grupo experimental executaram as técnicas e os conheci- mentos adquiridos durante a aplicação do programa. Por outra parte, os resultados observados no grupo-controle refletem que as mães que não recebem uma orientação específica mostram uma forte tendência ao desmame precoce. Nossos resultados de amamentação são comparáveis aos obtidos em outros estudos de aleitamento materno (e.g., Gulla \& Benech, 1984; Hardy et al., 1982; Martinic et al., 1985; Monteiro et al., 1987; Rea \& Cukier, 1988).

Em relação aos motivos alegados pelas mães para introdução de mamadeira, os mais mencionados foram: "pouco leite" e "o leite secou”, sendo que este último não apareceu no grupo experimental. Observando que os desmames aconteceram bem mais cedo no grupocontrole do que no grupo experimental, podemos dizer que a orientação durante o pré-natal é efetiva. Os dados indicam, no entanto, que esta orientação precisa ser contínua durante todo o período de amamentação, porque nos desmames entre quatro e seis meses que aconteceram no grupo experimental, as mães alegaram o primeiro dos motivos mencionados acima. Por outro lado, as respostas das entrevistas mostraram ser a indicação do pediatra a principal responsável pela introdução de mamadeira, tanto no grupo experimental (com 71,4\%) como no grupo-controle (com 55,6\%). Neste sentido, estudos anteriores já apontaram que os profissionais na área da saúde não são preparados adequadamente para dar apoio e instrução às mães que amamentam (Issler, 1987; Moura \& Santoro, 1980; Tudisco et al., 1984).

No que se refere à estimulação do bebê, os resultados refletem que as mães do grupo experimental utilizaram efetivamente as técnicas ensinadas no programa educacional. Os programas de estimulação precoce aplicados para populações de risco mostram que é possível obter resultados significativos no desenvolvimento destes bebês (Montenegro et al., 1977; Oliveira, 1979, 1982). Este último autor acrescenta que um nível pobre de estimulação recebido por crianças de famílias de baixo nível sócio-econômico é melhor preditor de risco para desnutrição severa do que o grau de pobreza em si mesmo. Vários outros autores (e.g., Affleck et al., 1982; Casto \& Mastropieri, 1986; Sameroff \& Fiese, 1990) chamaram a atenção também para os benefícios que os programas de intervenção precoce trazem tanto para crianças de alto risco como para aquelas portadoras de deficiências, sendo que esta intervenção será mais eficiente quando mais cedo ela for aplicada. Em particular, Casto e Mastropieri (1986), em sua meta-análise sobre a eficácia destes programas, destacam que estes be- 
nefícios consistiriam basicamente em ganhos no desenvolvimento cognitivo, de linguagem, motor e sócio-emocional.

Neste estudo não foi feita uma avaliação sistemática do desenvolvimento dos bebês, mas observações informais mostraram diferenças claras entre os bebês dos grupos experimental e controle. De modo geral, as observações indicaram que os bebês do grupo experimental apresentaram um desenvolvimento superior ao do grupo-controle em termos de vivacidade, balbuceio e agilidade, confirmando assim as previsões com base na literatura. Estas observações foram também corroboradas por comentários das próprias mães, como por exemplo: "meu bebê está muito esperto e diferente de meus filhos anteriores" (mãe do grupo experimental) e, por outro lado, "meu bebê é muito calmo, fica tranqüilo o tempo todo no berço, não dá trabalho" (mãe do grupo-controle). Comentários como este foram freqüentes nas mães do grupo-controle. Isto sugere que estas mães não estimulavam seus bebês por falta de conhecimento sobre a importância deste fator, e não se davam conta do atraso no desenvolvimento dos bebês e as suas implicações, chegando a considerar positiva a falta de desenvolvimento do bebê por implicar menos trabalho.

Investigamos também a existência de uma possível correlação entre o aleitamento materno e o número médio de ocorrências de estimulação. Fazendo um cruzamento entre estes dados verificamos que há um número maior de ocorrências de estimulação entre as mães que amamentavam seus filhos, em relação às que não os amamentavam (aproximadamente o dobro para as primeiras). Porém, ao fazer uma comparação entre os grupos, e considerando somente as mães amamentando, encontramos uma grande diferença no número médio de ocorrências de estimulação entre os grupos, sendo esta 2,5 e 4,8 vezes maior para o grupo experimental do que para o grupo-controle, no sétimo e oitavo registros, respectivamente. Em resumo, constatamos que: (1) as mães que amamentam estimulam mais seus bebês do que as mães que não amamentam, independentemente do grupo a que pertencem; e (2) as mães que receberam orientações e conhecimentos sobre a importância da interação mãe-criança efetivamente aplicaram as técnicas de estimulação ensinadas.

Desta maneira, nossos resultados evidenciam a importância de um programa de estimulação que enfatiza a interação mãe-criança para o desenvolvimento de bebês numa população considerada de risco.
A eficácia apresentada pelo programa educacional pode ser atribuída a vários motivos: 1) À formulação do programa educativo, com objetivos claros e com uma decomposição em objetivos intermediários, a fim de garantir a aprendizagem gradual dos conhecimentos e habilidades necessários tanto para aleitamento materno quanto para estimulação do bebê.

2) A uma metodologia com unidades de ensino, onde não se avançava para uma nova unidade enquanto não se verificasse a aquisição dos conhecimentos da unidade anterior.

3) À preparação prática das mães para utilizar as técnicas e superar as possíveis dificuldades que podem surgir durante o aleitamento materno. Na aplicação do programa educacional foi fundamental a utilização de recursos audiovisuais e do modelo prático ("seio cobaia”), e também a participação ativa por parte das mães, que não se limitavam a assistir passivamente às exposições, mas faziam intervenções a qualquer momento, relacionando o que estava sendo exposto com suas próprias experiências.

4) Às oportunidades que todas as participantes tiveram para realizar os exercícios na presença das colegas e da professora. Cada uma teve oportunidade de receber feedback das colegas e também de dar feedback sobre a execução dos exercícios por parte delas.

5) À freqüência de feedback positivo dado pela professora, quando da realização adequada dos exercícios. Além disso,ela fazia comentários e correções quando necessário.

Uma diferença fundamental entre este programa educacional e outros descritos na literatura é que, em geral, estes lidam exclusivamente com aleitamento materno ou com estimulação do bebê. O programa estudado nesta pesquisa trabalhou juntamente estes dois fatores, de tal modo que as mães podiam aproveitar a situação de aleitamento para estimular o bebê. A avaliação da orientação educacional fornecida às participantes foi feita através de observação direta, tanto do aleitamento quanto dos comportamentos de estimulação ensinados. Como as observações foram realizadas periodicamente por um tempo relativamente longo, o trabalho foi realizado com uma amostra pequena. Por este motivo, os resultados devem ser considerados ainda preliminares, mas indicam que a instrução conjunta sobre aleitamento materno e estimulação, para uma população de baixa renda, é viável e pode contribuir para a diminuição dos fatores de risco tanto para morbi-mortalidade e desnutrição quanto para atraso no desenvolvimento do bebê. 


\section{Agradecimentos}

Agradecemos ao Prof. Dr. Nivaldo Nale pela contribuição na elaboração do programa de ensino, e aos Profs. Benedito Galvão Benze e Lael Almeida de Oliveira, que colaboraram na análise estatística dos resultados deste trabalho. A primeira autora contou com bolsa de mestrado, e o segundo autor com bolsa de pesquisa, concedidas pelo CNPq.

\section{Referências}

AFFLECK, G.; McGRADE, B.; McQUEENEY, M. \& ALLEN, D., 1982. Relationship-focused early intervention in developmental disabilities. Exceptional Children, 49:259-261.

BOWLBY, J., 1976. El Vínculo Afectivo. Buenos Aires: Paidós.

BRAZELTON, T. B., 1981. Bebês e Mamães. Rio de Janeiro: Ed. Campus.

CAMPESTRINI, S., 1987. O "seio-cobaia" para ensinar como amamentar. Contact, 53 (encarte).

CASTO, G., \& MASTROPIERI, M., 1986. The efficacy of early intervention programs: a meta-analisis. $E x$ ceptional Children, 52:417-424.

GARCIA-MONTRONE, V., 1992. Avaliação de um programa educativo de incentivo ao aleitamento materno e estimulação precoce para mães de nível sócio-econômico baixo. Dissertação de Mestrado. São Carlos: Programa de Pós-Graduação em Educação Especial, Universidade Federal de São Carlos.

GRANZOTO, J.; BERTONI, A.; VECCHI, A. \& RODRIGUES, E., 1992. A importância do incentivo prénatal na amamentação de primíparas. Jornal de Pediatria, 68:34-37.

GULLA, G.; \& BENECH, C., 1984. Aleitamento materno e infecções em lactentes no Rio de Janeiro. Jornal de Pediatria, 57:430-433.

HARDY, E.; VICHI, A.; SARMENTO, R.; MOREIRA, L. \& BOSQUEIRO, C., 1982. Breastfeeding promotion: effect of an educational program in Brazil. Studies in Family Planning, 13:79-86.

HARDY, E.; SANDOVAL, L. \& PINOTTI, J., 1987. Aleitamento materno: um direito da mãe e da criança. Revista Paulista de Medicina, 105:103-107.

ISSLER, H., 1987. Aleitamento materno: dificuldades e propostas. Revista Brasileira de Medicina, 44: 282-283.

JUEZ, G., 1989. Lactancia materna: ventajas generales y nutricionales para el niño menor de un año. Revista Chilena de Pediatría, 60:3-8.

MARTINIC, J.; BRAVO, I.; \& PUGNO, R., 1985. Lactancia natural y nivel de salud. Boletín de la Oficina Sanitaria Panamericana, 98:548-556.

MARTINS FILHO, J., 1985. Interação alimentação-infecção na gênese da desnutrição infantil - Papel do leite humano. Jornal de Pediatria, 59:90-92.

MIURA, E., 1978. Leite materno, desnutrição e infecção. Revista da Associação Médica do Rio Grande do Sul, 22:8-15.

MONTEIRO, C.; ZUNINGA, H.; BENÍCIO, M. H. \& REA, M., 1987. Estudo das condições de saúde das crianças do Município de São Paulo - (Brasil), 19841985. III - Aleitamento Materno. Revista de Saúde Pública, 21:13-21.
MONTENEGRO, H.; LIRA, M. I. \& RODRIGUES, S., 1977. Early psichosocial stimulation program for infants (from birth to 24 months) of low socioeconomic class. In: Research to Practice in Mental Retardation. Care and Intervention. Volume I. (P. Mittler ed.) pp. 195-201. Baltimore: University Park Press.

MOURA, E. \& SANTORO, J., 1980. Aleitamento Materno II - Fatores de amamentação materna e desmame. Medicina, 12:21-29.

NETER, J. \& WASSERMAN, W., 1974. Applied Linear Statistical Models. Homewood, Illinois: Richard D. Irwin.

OLIVEIRA, A. J., 1979. A criança carente e a excepcionalidade. Revista Brasileira de Deficiência Mental, 14:37-43.

OLIVEIRA, A. J., 1982. A importância da estimulação programada sobre o desenvolvimento psicomotor de lactantes com marasmo nutricional. Re vista Brasileira de Deficiência Mental, 17:53-79.

OPS (Organización Panamericana de la Salud), 1988. Guia para la educación y participación comunitaria en el control del crescimiento y desarrollo del niño. Washington, DC: OPS.

REA, M. F. \& CUKIER, R., 1988. Razões de desmame e de introdução da mamadeira: uma abordagem alternativa para seu estudo. Revista de Saúde Pública, 22:184-191.

RODRIGUEZ-GARCIA, R. \& SCHAEFER, L. A., 1991. Nuevos conceptos de la lactancia, su promoción y la educación de los profesionales de la salud. Boletin de la Oficina Sanitaria Panamericana, 111:1-15.

SAMEROFF, A. \& FIESE, B. A., 1990. Transactional regulation and early intervention. In: Handbook of Early Childhood Intervention (Meisels, S. V.; Shonkoff, J. P., eds.), pp.119-149. Cambridge: Cambridge University Press.

SIQUEIRA, R.; DURSO, N.; ALMADA, A. G. P.; MOREIRA, M. T. \& MASSAD, G. B., 1994. Reflexões sobre as causas de desmame precoce observadas em dinâmicas de grupo de incentivo ao aleitamento materno. Jornal de Pediatria, 70:16-20.

TUDISCO, E. S.; MANOEL, N. DE J.; GOLDENBERG, P., NOVO, N. F. \& SIGULEM, D. M. 1984. Avaliação do estado nutricional materno e duração do aleitamento natural. Revista de Saúde Pública, 18: 313-322.

VICTORA, C. G.; SMITH, P. G.; VAUGHAN, J. P.; NOBRE, L. C.; LOMBARDI, C.; TEIXEIRA, A. M. B.; FUCHS, S. C.; MOREIRA, L. B.; GIGANTE, L. P. \& BARROS, F. C., 1989. Infant feeding and deaths due to diarrhea: a case-control study. American Journal of Epidemiology, 129:1032-1041.

VINHA, V. H. P., 1988. Projeto Aleitamento Materno: Determinação de sua eficácia com vistas ao autocuidado com a mama puerperal. Tese de Livre Docência. Ribeirão Preto: Escola de Enfermagem de Ribeirão Preto, Universidade de São Paulo.

VINHA, V. H. P. \& SCOCHI, C. G., 1989. Aleitamento Materno - Evolução Histórica. Revista Femina, outubro, 819-823.

WINDHOLZ, M. H., 1988. Passo a Passo, seu Caminho: Guia Curricular para o Ensino de Habilidades Básicas. São Paulo: Edicon. 